\title{
Graphene nanoribbon FETs for digital electronics: experiment and modeling
}

\author{
Kristof Tahy*, Huili Xing and Debdeep Jena* \\ Department of Electrical Engineering, University of Notre Dame, Notre Dame, IN 46556, USA
}

\begin{abstract}
One-dimensional nanostructures of graphene such as graphene nanoribbons (GNRs) can prove attractive for digital electronics in the form of interband tunneling transistors, as they are capable of high drive currents. Here, we report on the transport properties of p-n junctions formed in GNR field effect transistors (FETs). It is found that the current density in the devices is indeed high; in the $1-1.5 \mathrm{~A} / \mathrm{mm}$ range have been measured, comparable to Si-MOSFETs and III-V Nitride HEMTs. The observed unique current-voltage characteristics of the doublegated GNR FETs having a lateral p-n junction as their channel is explained by a field-effect model. Due to the lack of sufficiently large bandgap in the $30 \mathrm{~nm}$ wide GNR, the device still cannot be turned off completely, but rectification is achieved. The results suggest that the fabrication of tunneling FETs made out of graphene is possible and their characteristics may meet the expectations. Copyright (C) 2012 John Wiley \& Sons, Ltd.
\end{abstract}

Received 31 March 2011; Accepted 12 February 2012

KEY WORDS: graphene; nanoribbon; transistor; p-n junction; high-field transport

The recent discovery of graphene [1], a single atomic sheet of graphite, has ignited intense research activities to explore the electronic properties of this novel two-dimensional (2D) electronic system. Charge transport in graphene differs from that in conventional 2D electronic systems as a consequence of the linear energy dispersion relation near the charge neutrality (Dirac) point in the electronic band structure [2]. Though in theory, its electronic structure and transport properties are well known, the applicability as channel replacement material in conventional CMOS technology is still an open question. High mobility [3] and high current carrying capacity [4,5] make graphene very attractive, but on the other hand, low $\mathrm{I}_{\mathrm{ON}} / \mathrm{I}_{\mathrm{OFF}}$ ratio and the lack of sufficient saturation in $\mathrm{I}_{\mathrm{ON}}$ are yet unsolved drawbacks. In the past, we demonstrated these properties in double-gated graphene nanoribbon field effect transistors (GNR FETs). We successfully achieved $\mathrm{I}_{\mathrm{ON}} / \mathrm{I}_{\mathrm{OFF}}$ ratios of $10^{6}$ at cryogenic temperatures using either top or back gates, and sublinear output characteristic was observed [5]. Graphene has been studied mostly at low biases till date. For the application in practical devices, it is essential to investigate the high-field transport properties. In this letter, we present on the high field characteristics and the modeling of double-gated GNR devices biased partially n-type and partially p-type forming p-n junction such way.

Exfoliated graphene flakes on heavily n-type doped silicon wafers with $\mathrm{t}_{\mathrm{ox}}=300 \mathrm{~nm}$ thick thermal oxide from Graphene Industries were used for the experiments. The wafers were backside-metalized after oxide removal in buffered HF to form back gate contacts. The graphene flakes were patterned successfully by $\mathrm{O}_{2}$ plasma reactive ion etch forming nanoribbons with widths down to $20 \mathrm{~nm}$ [6]. To achieve this, $20 \mathrm{~nm}$ thick $\mathrm{Al}$ as a mask was used patterned by e-beam lithography using PMMA and lift-off. $\mathrm{Cr} / \mathrm{Au}$ source/drain contacts and $\mathrm{Al}_{2} \mathrm{O}_{3} / \mathrm{Ti} / \mathrm{Au}$ as top gate $\left(\mathrm{t}_{\mathrm{ox}}=30 \mathrm{~nm}\right)$ have been

*Correspondence to: Kristof Tahy and Debdeep Jena, Department of Electrical Engineering, University of Notre Dame, Notre Dame, IN 46556, USA.

E-mail:djena@nd.edu 
deposited to form FETs. After the source/drain metal deposition and lift-off, the samples were annealed in forming gas at $\sim 400^{\circ} \mathrm{C}$ for $\sim 2 \mathrm{~h}$ to remove the e-beam resist residue [7]. $1 \mathrm{~nm}$ e-beam deposited $\mathrm{Al}$ was used as seed layer for the ALD-deposited $\mathrm{Al}_{2} \mathrm{O}_{3}$. The channel length of the fabricated devices is $2 \mu \mathrm{m}$, and the length of the top gate electrode is about $1 \mu \mathrm{m}$, covering half of the channel.

The SEM image in Figure 1 (a) shows a typical GNR FET, and a schematic cross section can be seen on Figure 1 (b). High current annealing [8] was performed to drive off impurities for some FETs to recover their intrinsic performance. The devices were measured using a semiconductor parameter analyzer in ambient environment and in vacuum $\left(5 \times 10^{-5}\right.$ Torr $)$, at room temperature at $77 \mathrm{~K}$ and at $4 \mathrm{~K}$.

In this paper, we present the measurements of a single device with $30 \mathrm{~nm}$ GNR width at room temperature and on $4 \mathrm{~K}$. It is found that at high applied source-drain current, the temperature has very little effect on the device performance, namely at cryogenic temperatures, the high-field modulation is only $\sim 10 \%$ more. We fabricated many similar devices, and we observed similar characteristics in all cases. Figure 2 (a) and (b) shows the source-drain conductance at $\mathrm{V}_{\mathrm{DS}}=20 \mathrm{mV}$ and at $\mathrm{V}_{\mathrm{DS}}=1 \mathrm{~V}$, respectively, as a function of the top gate and the back gate. The back gate capacitance is $\sim 11 \mathrm{nF} / \mathrm{cm}^{2}$ assuming $\kappa=3.9$, and the top gate capacitance is $209 \mathrm{nF} / \mathrm{cm}^{2}$ calculated based on the measurement of the minimum conductance (Dirac) point as shown in Figure 2 (b). The Dirac point is defined by the ratio of the applied voltages on both gate, the slope defining the value of $C_{\text {top }} / C_{\text {back }} \sim 19$. From both a constant back gate or constant top gate slice, we can determine $\mathrm{V}_{\mathrm{TG}}^{0}=-5 \mathrm{~V}$ and $\mathrm{V}_{\mathrm{BG}}^{0}=-45 \mathrm{~V}$,

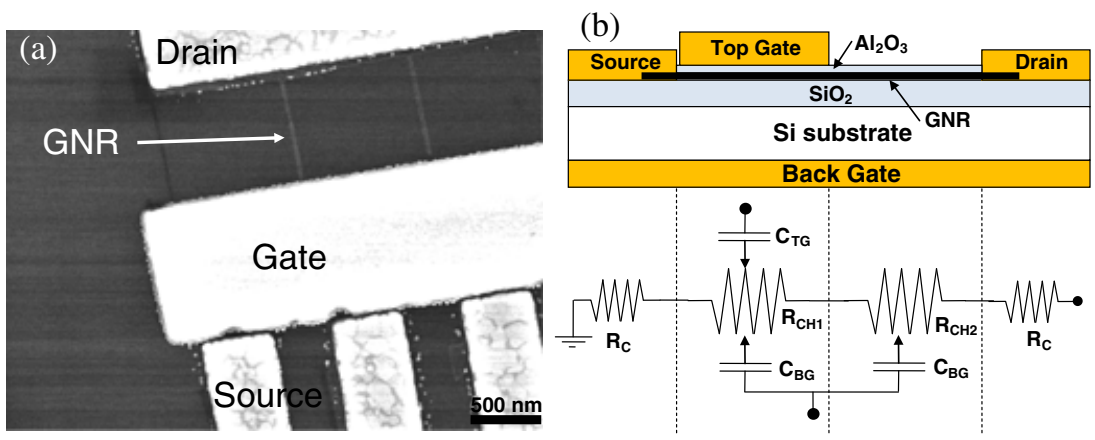

Figure 1. (a) SEM micrograph of GNR FETs. Half of the channels are top gated, while the whole device is back gated. The channel length is $2 \mu \mathrm{m}$, the gate width is $1 \mu \mathrm{m}$. The width of the GNR is $30 \mathrm{~nm}$. (b) Device schematics and wiring diagram of the device model showing the critical resistances and the influence of the gates on the two parts of the channel.

(a)

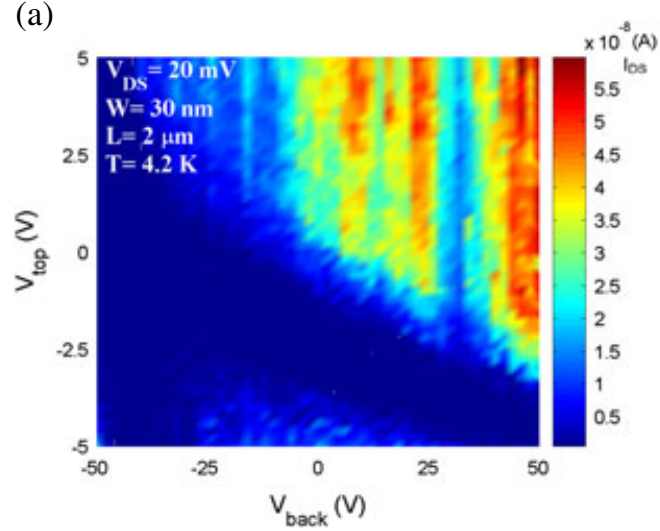

(b)

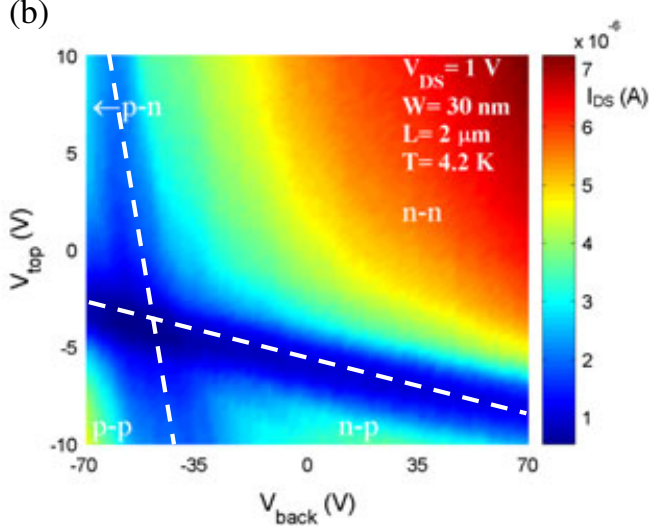

Figure 2. (a) Conductance steps at $4.2 \mathrm{~K}$ at low source-drain voltage (linear scale); (b) At higher bias ( $\left.\mathrm{V}_{\mathrm{DS}}=1 \mathrm{~V}\right)$, the modulation is smaller. This high bias conductance is not influenced by the temperature; room temperature measurement yields the same result. 
indicating strong n-type doping. While the devices before the top gate deposition were slightly p-type doped, we can conclude that the strong n-doping is due to trapped charges in the top gate oxide and other impurities on the graphene oxide interface. A comparison of the transfer characteristics before and after the top gate oxide deposition can be seen on Figure 3 (a) and (b).

Temperature-dependent measurements of the off-state conductance have confirmed bandgap opening of $>26 \mathrm{meV}$ depending on GNR widths and result in $20 \mathrm{x}$ and $10^{3} \mathrm{x}$ modulation at room temperature and $4 \mathrm{~K}$, respectively, by varying the top gate potential between $+/-5 \mathrm{~V}$ (Figure 3 ). Quantized conductance was observed at low temperature, which is clearly indicates that the carriers in the GNR channel are one dimensionally confined as observed before [9]. This phenomenon also gives us a tool to extract the value of the GNR bandgap; details of these measurements and modeling are detailed in an earlier report [6].

The source and drain contacts are in touch with a large 2D graphene region to ensure low contact resistance. The 2D graphene has no bandgap so the $\mathrm{Cr} / \mathrm{Au}$ metal can form good ohmic contacts easily. The back gate may vary the carrier concentration of the graphene at the contacts, but due to its large size, it will have always much lower resistance than the GNR channel, always supplying the channel with high enough number of carriers.

Figure 4 (a), (b), and (c) shows the measured $\mathrm{I}_{\mathrm{DS}}$ for different top gate voltages at $\mathrm{V}_{\text {back }}=-70 \mathrm{~V}, 0 \mathrm{~V}$, and $+70 \mathrm{~V}$, respectively. To understand the device operation, we have to examine the device structure in detail. The channel of the devices can be separated into two distinct regions: one having back gate only and the other having both top and back gates. A model has been developed to explain the transistor characteristics of the p-n channel GNR FET by extending the work of Meric et al. [4].

We applied a field effect model to both sides of the junction separately. The applied gate and source-drain voltages determine the carrier concentration on both parts of the channel according
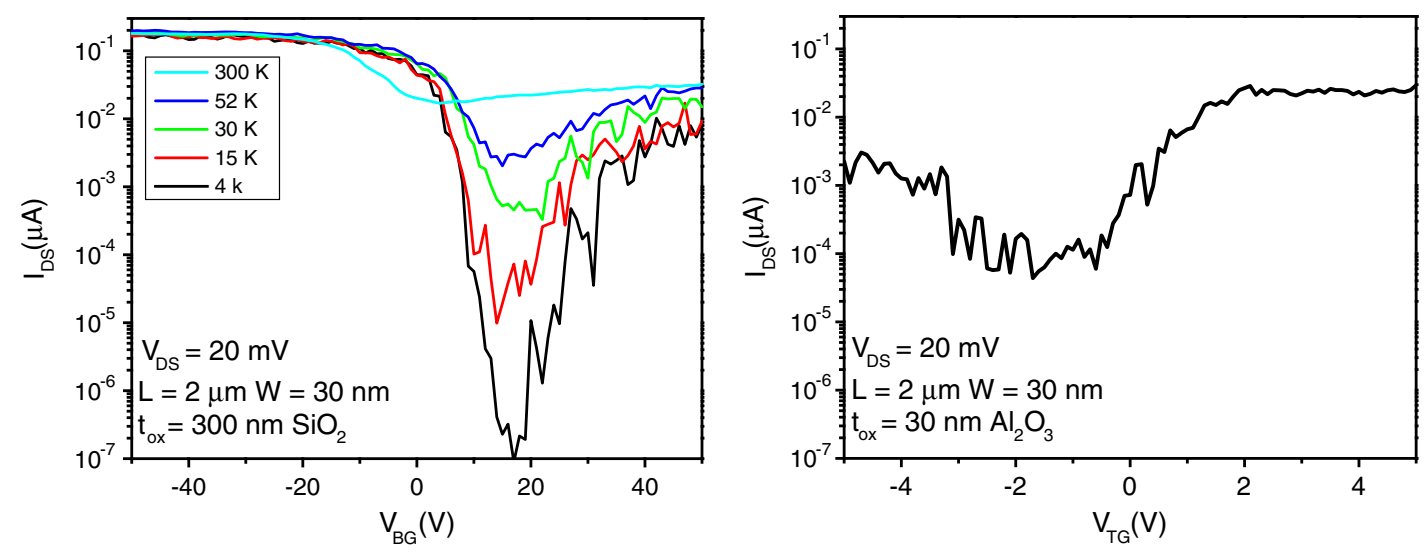

Figure 3. Comparison of the transfer characteristics (a) before and (b) after the top gate oxide deposition. The initially p-doped device shifted to $n$-doped. The $\mathrm{I}_{\mathrm{ON}} / \mathrm{I}_{\mathrm{OFF}}$ ratio decreased by several orders of magnitude. (a) shows the temperature dependence of $\mathrm{I}_{\mathrm{DS}}$, which is used as the base of the bandgap extraction.
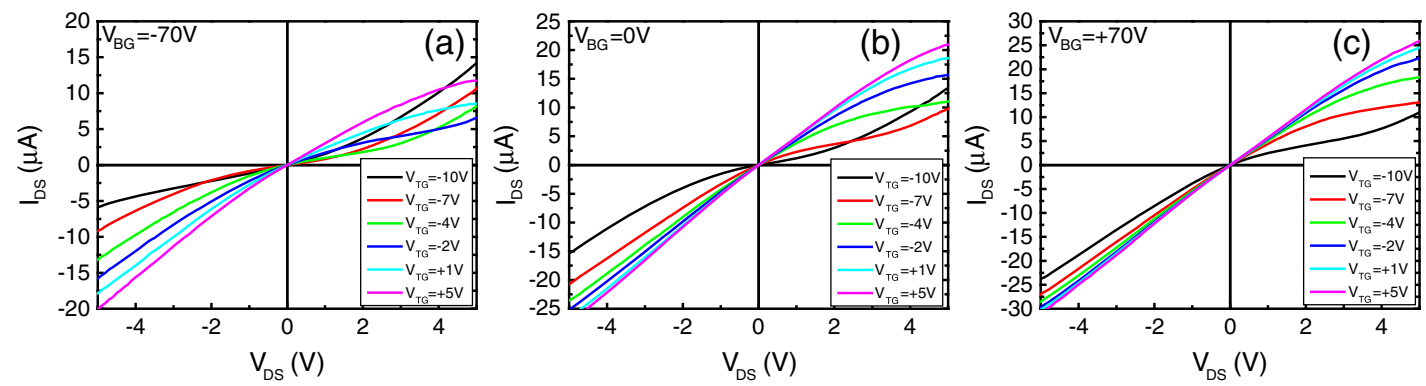

Figure 4. Measured room temperature $\mathrm{I}_{\mathrm{DS}}$ versus $\mathrm{V}_{\mathrm{DS}}$ at $-70 \mathrm{~V}(\mathrm{a}), 0 \mathrm{~V}(\mathrm{~b})$, and $+70 \mathrm{~V}$ (c) back gate bias. 

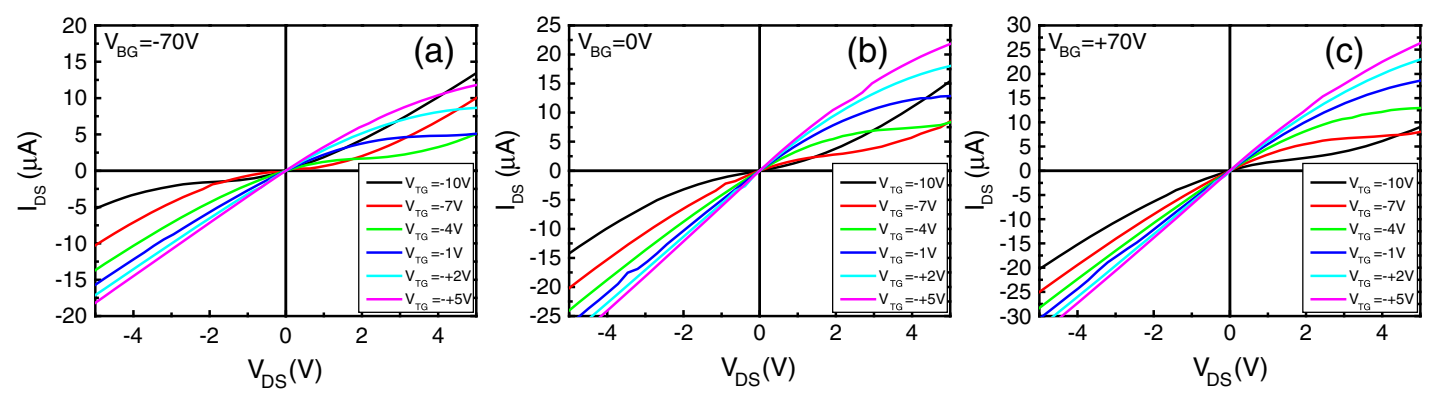

Figure 5. Simulated device characteristic at the same bias conditions as the device shown in Figures 3 and 4. $\mathrm{I}_{\mathrm{DS}}$ versus $\mathrm{V}_{\mathrm{DS}}$ at $-70 \mathrm{~V}(\mathrm{a}), 0 \mathrm{~V}(\mathrm{~b})$, and $+70 \mathrm{~V}$ (c) back gate bias.

$$
n(x)=\sqrt{n_{0}^{2}+\left(\frac{C_{t g}}{q}\left(V_{t g}-V_{t g 0}-V(x)\right)+\frac{C_{b g}}{q}\left(V_{b g}-V_{b g 0}-V(x)\right)\right)^{2}}
$$

where $x$ is the distance along the channel and $V(x)$ is the potential in the channel due to the applied source-drain voltage. A schematic of the model is shown on Figure 1 (b). The current in the channel is expressed by

$$
J(x)=q n(x) \mu F(x)
$$

where $\mu$ is the mobility and $F(x)$ is the electric field along the channel. The I-V characteristics are obtained by forcing current continuity in a self-consistent electrostatic and transport model

$$
I\left(V_{D S}\right)=q \frac{W}{L} \mu \int_{0}^{V_{D S}} n(x) d V(x)
$$

where $L$ is the channel length, and $W$ is the GNR width. Carrier drift velocity and mobility saturation are considered depending on the carrier concentration based on the work of Dorgan et al. [10].

$$
v_{s a t}=\frac{\omega_{o p}}{\sqrt{\pi n(x)}}
$$

where $\omega_{o p}$ is the optical phonon wavelength of the dominant scattering phonons, which are surface optical phonons of the high- $\kappa$ oxide. And

$$
\mu=\frac{\mu_{0}}{\sqrt{1+\left(\frac{\mu_{0} V_{D S}}{L v_{s a t}}\right)^{2}}} .
$$

For simplicity, we assumed equal mobility and saturation velocity to describe both the electrons and holes. The solution not only provides the I-V characteristics of the device but detailed information about the carrier concentration under various bias conditions. Figures 4 and 5 demonstrate the experimental and modeling data, showing close agreement. As input parameters in the model, we used mobility of $300 \mathrm{~cm}^{2} / \mathrm{Vs}$, a minimum carrier density of $5 \cdot 10^{11} \mathrm{~cm}^{-2}$, and source-drain series resistance of $0.5 \Omega \mathrm{mm}$. The saturation of current at high positive bias is caused by depletion of the carriers even in those cases when the gate biases set a high initial carrier concentration. At high negative drain bias, on the other hand, lack of saturation is observed. This is because the drain bias in this case further increases the carrier concentration instead of depleting. Depending on the gate bias conditions, the flattening of the current occurs at different applied source-drain bias. If 
$\mathrm{V}_{\mathrm{BG}}=-70 \mathrm{~V}$ ((a) on Figures 3 and 4$)$ as $\mathrm{V}_{\mathrm{TG}}$ increases, one can see that plateau of the $\mathrm{I}_{\mathrm{DS}}$ occurs at higher and higher positive bias. At positive $\mathrm{V}_{\mathrm{TG}}$ pinch-off occurs at $\mathrm{V}_{\mathrm{DS}}=5 \mathrm{~V}$ only. At more positive back gate biases ((b) and (c) on Figures 3 and 4), the plateauing of the current occurs at highly negative top gate biases only. Superlinear current increase is caused together by the sharp increase of carrier concentration close to the Dirac point and the increase of the accelerating field.

In conclusion, p-n junction in GNR FETs has been experimentally demonstrated. The analysis of the device operation shows that sublinear and superlinear features observed in the I-V characteristics are due to the device electrostatics. The p-n junction channels are practically transparent to interband tunneling of carriers due to the small bandgap and high applied bias but offer an exciting novel structure for configuring and designing device characteristics with various functionalities.

\section{ACKNOWLEDGEMENTS}

This work was supported by the Semiconductor Research Corporation Nanoelectronics Research Initiative and the National Institute of Standards and Technology through the Midwest Institute for Nanoelectronics Discovery.

\section{REFERENCES}

1. Novoselov KS, Geim AK, Morozov SV, Jiang D, Zhang Y, Dubonos SV, Grigorieva IV, Firsov AA. Science 2004; 306:666.

2. Zhang Y, Tan Y-W, Stormer HL, Kim P. Nature 2005; 438:201.

3. Geim AK, Novoselov KS. Nature Materials 2007; 6:183.

4. Meric I, Han MY, Young AF, Oezyilmaz B, Kim P, Shepard KL. Nature Nanotechnology 2008; 3:654.

5. Tahy K, Koswatta S, Fang T, Zheng Q, Xing H, Jena D. IEEE Device Research Conference Digest 2009; 67:207.

6. Lian C, Tahy K, Fang T, Li G, Xing H, Jena D. Applied Physics Letters 2010; 96:103109.

7. Ishigami M, Chen JH, Cullen WG, Fuhrer MS, Williams ED. Nano Letters 2007; 7:1643.

8. Moser J, Barreiro A, Bachtold A. Applied Physics Letters 2007; 91:163513.

9. Lin Y, Perebeinos V, Chen Z, Avouris P. Physical Review B 2008; 78:161409.

10. Dorgan V, Bae M-H, Pop E. IEEE Device Research Conference Digest 2010; 68:73. 